\title{
Conditions didactiques de la transformation d'une liste de diffusion en outil collaboratif
}

\author{
Stéphane Simonian* — Jean Ravestein* — Jacques Audran** \\ * Université de Provence \\ Département des Sciences de l'Education \\ 1, avenue de Verdun F-13410 Lambesc \\ s.simonian@educaix.com ; jean.ravestein@up.univ-mrs.fr \\ *** Université de Haute-Alsace \\ Cufef, 17, rue Alfred Werner F-68098 Mulhouse \\ jacques.audran@uha.fr
}

RÉSUMÉ. Cette recherche analyse les échanges par Emails capturés sur une liste de diffusion utilisée par des étudiants inscrits en MASTER «Ingénierie de Formation et Multimédia » de l'Université de Provence (France). Dans ce cursus, cette liste a pour fonction de permettre aux étudiants de communiquer pour produire des connaissances et non pour simplement s'entre-aider à restituer correctement des contenus académiques. Notre hypothèse est qu'un changement de logique au niveau des buts pédagogiques favorise la transformation d'une liste de diffusion en véritable outil collaboratif. Notre analyse informatisée du corpus montre qu'inviter les étudiants à construire des connaissances (activité de production et non de restitution) détermine les types d'échange entre apprenants. Ainsi, si travailler dans un environnement collaboratif permet d'échanger des ressources, de partager des contenus numériques mais aussi des idées et des réflexions, il devient davantage un véritable outil au service de l'apprentissage lorsque le but de l'activité commune est la réalisation d'un projet concret.

ABSTRACT. This research analyzes a corpus or emails from a mailing list used by master students at the University of Provence (France) ("Engineering of Formation and Multimédia" programme). This list is intended to support knowledge production and not simply to help the students do academic homework. Our hypothesis is that this paradigm shift transforms the mailing list into a real collaborative tool. A computerised analysis of the corpus shows that inviting students to build knowledge determines the types of exchange between them, in particular if the task to be accomplished is oriented towards a concrete product. Working in a collaborative environment allows students to exchange resources, to share numeric contents as well as ideas and reflections. It therefore becomes more a real tool for training when the goal of the common activity is the achievement of a concrete project.

MOTS-CLÉS: liste de diffusion, environnement collaboratif, production de connaissances, interaction humaine, échanges asynchrones.

KEYWORDS: mailing list, collaborative environment, knowledge production, human interaction, asynchronous exchanges.

Distances et savoirs. Volume $4-n^{\circ}$ 4/2006, pages 513 à 526 


\section{Introduction}

Cette recherche se situe dans le champ de l'étude des interactions homme-homme lorsqu'ils utilisent des machines pour communiquer (Barchechath et Pouts-Lajus, 1990 ; Linard, 2000). Ce que nous appelons interaction ne nécessite pas forcément l'utilisation de machines dites « interactives », et, inversement, les ordinateurs ne conduisent pas à créer de l'interaction. En effet, si l'interactivité est une notion d'origine technique, l'interaction est une notion plus ancienne issue de la psychologie sociale. L'interactivité peut être intentionnelle (Jacquinot, 1996) et vue comme une relation simulée de communication entre l'auteur, l'émetteur, et l'usager ${ }^{1}$; il semble nécessaire de la distinguer de l'interaction qui, elle, est humaine et concerne « simplement » l'interdépendance et l'influence réciproques des individus entre eux. Cette distinction permet de définir un cadre théorique et méthodologique propre aux situations communication médiées par ordinateur en utilisant des analyses socio-discursives. Elle offre également l'opportunité d'établir une distinction du même type entre la «médiatisation $»^{2}$ et la « médiation $»^{3}$ pédagogique. En d'autres termes, nous souscrivons au point de vue de Linard, (2000) qui refuse de réduire les relations enseignant-apprenant ou apprenant(s)-apprenant(s) à des opérations d'« automates » ou à des systèmes purement logiques. C'est donc dans le cadre de l'interaction humaine que nous nous proposons d'examiner des échanges écrits asynchrones entre apprenants via une liste de diffusion créée à l'occasion de leur participation à un diplôme de Master Professionnel ${ }^{4}$.

Cette recherche se situe également dans le champ de la didactique dans la mesure où une idée directrice est de mettre en évidence que des échanges écrits asynchrones entre apprenants portent pour une grande part sur des difficultés relatives à l'appropriation de savoirs proposés dans le cursus, au moins autant que sur des questions personnelles ou périphériques à la formation. Notre liste de diffusion, qui permet et même invite les étudiants à "produire» pour des pairs du savoir sur les savoirs et les fait par là même sortir d'une simple logique de « restitution » des connaissances à destination d'une institution qui évalue, peut prendre le statut d'outil collaboratif au service des apprentissages. Passer d'une ingénierie didactique souvent arc-boutée sur la restitution normée des connaissances à une ingénierie qui fait la part belle à l'intervention de l'apprenant sur la constitution même des savoirs nous semble un pari pédagogique intéressant. L'évolution récente du $W E B$ vers le $W E B$ 2.0, dans laquelle on remarque une intervention spontanée de plus en plus importante des utilisateurs sur les contenus en terme d'avis ou même de contributions nous encourage à penser que les mentalités évoluent vis-à-vis du rapport au savoir

1. La différence avec l'interactivité machinique (homme-machine) est que cette dernière dépend essentiellement de la réactivité d'un dispositif qui permet de répondre adéquatement et qui tente de s'adapter à l'utilisateur (Jacquinot, 1996 ; Peraya, 1998).

2. Approche technocentrée.

3. Approche anthropcentrée.

4. Master 2 «Education et Formation », parcours «Ingénierie de Formation et Multimédia » Université de Provence. Sciences de l'Education. 
dans la société et que les formations utilisant des outils collaboratifs doivent mettre à profit ces nouvelles «attitudes", en particulier en fédérant les énergies des étudiants autour de projets concrets.

\section{Le contexte de la recherche et le cadrage théorique}

Pour reprendre la typologie d'Henri et Pudelko (2002), la communauté sur laquelle nous centrons notre recherche est constituée de manière occasionnelle autour d'une tâche prescrite ; elle a une durée de vie limitée (8 mois) liée au calendrier général de la formation et à l'échéancier particulier de la tâche. Dans notre contexte, les échanges entre apprenants grâce à une liste de diffusion ne constituent ni une obligation ni un enjeu d'évaluation. Cette recherche se situe donc dans le champ des environnements collaboratifs (Slavin, 1977 ; Frye et Coe, 1980 ; Hoyles, Healy et Pozzi, 1992, 1994 ; Kopper, 2003 ; Dubour et al., 2005), notamment à travers des modèles socio-constructivistes (Johnson, Johnson et Stanne, 1986 ; Viens et al., 2001 ; Henri et Lundgren-Kayrol, 2001).

Nous tenterons d'apporter des éléments de compréhension du processus collaboratif en faisant l'hypothèse qu'il modifie favorablement le rapport aux connaissances à condition que l'activité proposée aux apprenants soit orientée vers une production (Ravestein, 2006) et qu'il contribue ainsi à la qualité de l'apprentissage (Harasim et al., 1995 ; Bottino, 2004). Notre objectif est de mieux comprendre les finalités de l'usage des listes de diffusion dans une «institution didactique » (Sensevy, 1998) : dans quelles situations didactiques sont-elles une aide à l'apprentissage ? Quels types d'échanges sont alors privilégiés ? La liste de diffusion devient-elle une véritable ressource (Laurillard, 1995) entendue d'une manière générale comme émanant de la mutualisation et du partage d'unités de savoirs ; mais aussi, dans le contexte d'un travail collaboratif, d'échange de points de vue, d'idées, de doutes, de questions?

Nous adoptons dans ce travail un parti pris empirique qui favorise une analyse située, sensible aux spécificités des groupes et des contextes, attentive au problème de la distinction entre les pratiques qui ne font que reproduire avec de nouveaux médias des manières de faire traditionnelles et de nouvelles pratiques émergentes qui s'ajustent aux technologies employées, à leurs contraintes et à leurs possibilités, à leurs contextes d'usage spécifiques (Mondada, 1999).

Pour le chercheur, l'avantage méthodologique des outils comme le forum ou la liste de diffusion est qu'ils rendent possible la capture des messages et l'identification de l'objet $a$ priori $^{6}$ choisi par l'émetteur. Ainsi, les échanges asynchrones deviennent

5. Il s'agit de réaliser en petits groupes, constitués par centre d'intérêt communs des participants, un produit multimédia censé contribuer à l'amélioration de la formation qu'ils reçoivent.

6. Renseignement de la rubrique «thème» ou «sujet» dans les logiciels de gestion de courriels. 
des objets qui peuvent êtres manipulés (Henri et Lundgren-Kayrol, 2001). Les traces des discours au sein des archives sont ainsi facilement récupérées et peuvent être triées et analysées.

Toutefois, les échanges asynchrones sont soumis à certaines contraintes : l'absence d'indices paraverbaux peut engendrer des difficultés de compréhension des messages, même lorsqu'on en connaît le contexte d'énonciation. En revanche, l'immédiateté de la communication ainsi que l'accessibilité élargie aux ressources constituent des avantages des environnements informatiques d'apprentissage (Zack, 1995 ; Myrdal, 1994).

Le modèle de l'apprentissage expérientiel propose un cadre théorique pour mieux comprendre les effets de l'absence de médiation humaine in vivo dans le contexte de la formation à distance (Lamirault 2002).

Comme nous le rappelle Kaye (1992), collaborer signifie travailler ensemble avec une intention explicite d'ajouter de la valeur, ce qui implique de partager des buts et, de créer quelque chose de nouveau ou de différent grâce à la collaboration. Collaborer se distingue donc de l'échange simple d'information ou de transmission d'instruction. Une autre caractéristique se situe dans la répartition du travail (Teasley et Roschelle, 1993). Le travail collaboratif passe, entre autres, par un engagement mutuel des participants pour résoudre un problème où chaque individu est en partie responsable d'une partie de la résolution.

Si on suit Engeström (1987), qui s'appuie sur les travaux de Bateson (1974), l'activité individuelle et collective dépend des instruments utilisés, sujets, objets, division du travail, règles appliquées et communauté. Il nous invite à adopter un point de vue non exclusivement fondé sur la communauté ou les instruments, mais prend en compte l'ensemble du contexte des activités. Engeström (1992) distingue aussi la coordination (chacun se contente d'exécuter son rôle et actions) de la coopération (problème commun dans lequel chaque membre tente de trouver des façons mutuellement acceptables de le conceptualiser) et de la communication réflexive (conceptualiser ses propres interactions en relation avec les objets d'activités partagés). Pour cet auteur, ces trois phases constituent un cycle naturel de toute activité d'apprentissage. Ainsi, in concreto, l'activité d'apprentissage peut être conceptualisée comme une pratique sociale à travers la notion de « construction collaborative des connaissances » (Stahl, 2002).

En fait, la liste de diffusion d'un groupe restreint est un objet « vivant », une sorte de corps complexe (Choplin et al., 2005), mais dans lequel nous ne désespérons pas de débusquer une certaine logique de construction et de fonctionnement. Cette logique se manifesterait pour nous, par hypothèse, à travers la proportion très importante d'échanges portant sur la régulation de la réalisation de la tâche de production prescrite. 


\section{Objet de la recherche}

Lors des précédents travaux (Audran et Simonian, 2003 ; Simonian et Ciussi, 2004) nous avons proposé une grille de lecture des échanges pour un groupe d'apprenants en différenciant les échanges liés à la technique de ceux de compréhension de contenu et d'évaluation. Ces premiers travaux concernaient la restitution d'une tâche et non la production de savoirs. Cette dernière activité revêt pour nous un caractère spécifique qui peut différencier les types d'échanges. Nous alors avons capturé les échanges sur une liste de diffusion durant 9 mois de formation entre les 15 étudiants d'un diplôme de master et leur tuteur, avec leur assentiment. Le corpus de la liste étudiée ici comporte 629 messages capturés entre octobre 2003 et juin 2004. Même lorsqu'on ne compte pas les semaines de congés ou les semaines où le groupe se rencontre pour travailler dans un même lieu, les émissions de messages présentent un profil particulier : durant les 5 premières semaines l'irrégularité est la plus grande (de 63 messages à 0 message); avec un moyenne hebdomadaire d'émission de 15 messages les 10 semaines suivantes l'activité est sensiblement en baisse ; une plus grande régularité de l'activité apparaît à partir de la $15^{\mathrm{e}}$ semaine avec un phénomène d'alternance et 23 messages de moyenne, et finit à 10 messages de moyenne les 10 dernières semaines.

\section{Messages par semaine}

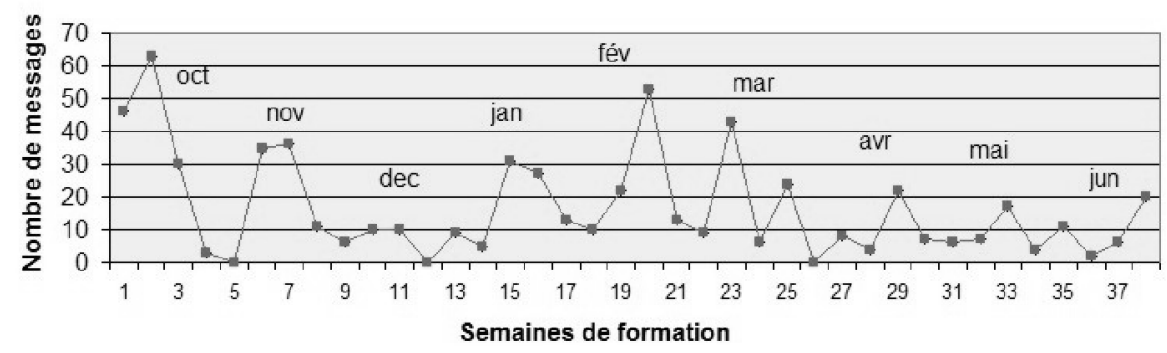

Graphique 1. Quantité de messages par semaines de formation

Le graphique rend compte d'une activité oscillante faisant chevaucher deux périodes cycliques ( 1 période de 4 semaines, et une période de 18 semaines). Les périodes s'observent à partir des modes globalement constants : 8 modes mensuels (à l'exception de décembre, période traditionnellement consacrée aux congés) et 2 modes semestriels en octobre et janvier correspondant respectivement au début de la formation (début de l'année universitaire) et au début de l'année civile. Une tentative d'explication peut tenir au fait que dans cette formation les étudiants se retrouvent dans un même lieu toutes les 4 semaines. L'oscillation régulière entre les maxima et les minima qui transparaît dans le rythme d'émission des messages s'effectue tout particulièrement dès la $15^{\mathrm{e}}$ semaine, quand la formation a pris son rythme de croisière. Cette alternance est alors bien visible jusqu'à la fin de la formation. Sur cette durée, 
la semaine de regroupement, et celle qui suit immédiatement, sont toujours celles qui donnent lieu au nombre maximal d'échanges. L'émission des messages, leur régularité cyclique et leur maintien apparaissent donc intimement liés à la présence et à la rencontre. L'absence, contrairement à ce qu'on pourrait attendre, n'engendre pas une augmentation des messages, bien au contraire. Ces points nous paraissent mettre en évidence l'importance de l'interaction dans les échanges verbaux écrits, même quand ils se font au moyen de machines à communiquer.

Nous souhaitons mettre en évidence, dans des échanges par liste de diffusion, l'importance respective de deux types de logique d'apprentissage: un premier type basé sur la « restitution » correcte des contenus à travers un contrôle par des exercices a fait l'objet de recherches antérieures ; ici nous étudions un second type d'apprentissage fondé sur la "production» de savoirs à partir des contenus de la formation pour réaliser une tâche (Ravestein, 2006). Les précédents travaux que nous avons publiés étaient centrés sur la constitution des groupes et des modes d'échanges dans une logique de "restitution» (Ravestein et Simonian, 2003 ; Audran et Simonian, 2003 ; Simonian et Ciussi, 2004). Ces recherches nous ont conduit à analyser les échanges entre apprenant(s)-apprenant(s) et apprenant(s)tuteurs selon les catégories suivantes :

- la technicité : les échanges concernant des difficultés d'accès à des contenus ;

- la compréhension : les échanges relatifs à l'intégration de contenus ;

- l'évaluation : les échanges évoquant les consignes et les modalités de validation des acquis.

Ces trois catégories peuvent être considérées comme des « indicateurs graduels » des profils des apprenants en fonction de leur parcours d'apprentissage et de l'avancée dans leurs compétences globales. L'analyse d'un premier corpus (Audran et Simonian, 2003) a montré qu'une fois les questions techniques écartées, ce sont les échanges " compréhension de contenu » qui prédominent sur ceux concernant l'évaluation. L'analyse d'un deuxième corpus a mis en évidence la nécessité d'ajouter une catégorie supplémentaire que nous avons nommé «lien social » (Simonian et Ciussi, 2004). En effet, constituant $13 \%$ des échanges, elle est un indice de reliance entre les apprenants, reflète l'enthousiasme des élèves et leur engagement dans le projet.

L'analyse du corpus de messages de la présente recherche s'appuiera au départ sur cette catégorisation, tout en nous laissant l'opportunité de la dépasser et de faire émerger d'autres catégories par l'utilisation de logiciels de traitement de corpus langagiers. Pour étudier notre corpus de textes nous serons également aidés par la distinction opérée par Audran (2001, 2000, 2001) entre la dynamique verticale des échanges centrée sur l'information (un membre fait part d'une information) et celle, horizontale, orientée vers la discussion (un membre propose un sujet de discussion). En première approche, nous faisons l'hypothèse selon laquelle une logique de restitution favorise une dynamique verticale, alors qu'une situation d'apprentissage axée sur une logique de production favorise une dynamique horizontale, qui comme 
on l'a vu peut laisser apparaître des tendances cycliques liées aux moments de rencontre.

Cependant, tout comme les résultats des deux premières analyses (Audran et Simonian, 2003 ; Simonian et Ciussi, 2004), ceux que nous allons présenter sont limités par le fait que les outils communicationnels institutionnalisés ne génèrent pas forcément des échanges longs et riches et que le mode d'échange prédominant est celui de la « question-réponse » (Henri et Pudelko, 2002). Nous devons tenir compte également du fait que la prise de parole sur une liste de diffusion est très concentrée par une minorité active (Drot-Delange, 2001 ; Audran, 2002 ; Audran et Simonian, 2003). Enfin, la limite principale de notre analyse est d'ordre méthodologique.

En effet, l'analyse des échanges ne s'effectuera pas en fonction des interactions entre les apprenants, mais sur les unités de sens (lemmes). Ainsi, nous ignorerons les adressages (qui dit quoi à qui ?) car nous voulons travailler sur la fréquence des termes employés à l'occasion d'une tâche de production indépendamment de la problématique émetteur-récepteur. Cette prise de position méthodologique permet d'articuler les analyses effectuées sur les discours des étudiants dans cette recherche (logique de production) avec celles de nos précédents travaux (logique de restitution). Le fait de travailler sur des lemmes permet également de tenir compte du discours global effectué par un groupe d'étudiants en mettant surtout l'accent sur le type d'échange. Comme l'indique Lipponen (2002), il n'y a pas encore de cadre théorique établi et unifié pour analyser des échanges par listes de diffusion. Ainsi, pour l'analyse, nous retiendrons les travaux sur la granularité du discours, notamment les trois dimensions proposées par Peraya $(2000,2002)$ : référentielle, relationnelle, régulatrice. Nous reprendrons également les deux axes (vertical et horizontal) mis en avant par Audran (2001) et les catégories de nos recherches précédentes (technique, compréhension, évaluation et lien social). Nous utiliserons donc un cadre d'analyse et des catégories ad hoc pour notre corpus en tenant compte des propositions précédentes et de nos travaux antérieurs.

\section{Corpus, méthode d'analyse et résultat}

De manière très générale, lorsqu'on se propose d'analyser les échanges sociaux sous leur aspect langagier, un problème important tient à la manière d'isoler et de formaliser les fils de discussion (threads). Goffman (1987) relève que ces fils ne sont pas aussi linéaires que l'on pourrait le penser. Le dialogue serait à « plusieurs voix » (échange avec des simultanéités, c'est-à-dire des discussions parallèles) réalisé par des phases successives. Les fils de discussion forment ainsi des réseaux relativement difficiles à décrire (Audran, 2001, 267).

De manière plus spécifique, l'analyse détaillée de l'aspect séquentiel des discours asynchrones échangés par listes de diffusion touche à des problèmes tels que :

- la manière dont les processus de production et de compréhension sont rendus manifestes (autant aux locuteurs qu'à l'analyste) ; 
- la façon dont l'intersubjectivité est défendue, maintenue, rétablie ;

- la manière dont l'espace interactif est configuré par la structure des échanges ;

- la gestion des échanges polémiques, conflictuels, manifestant des désaccords profonds.

En effet, alors que majoritairement nos étudiants travaillaient jusqu'en licence (bachelor degree) dans une logique de restitution des connaissances (cours, TD, examens), on les place en Master devant la nécessité de produire des connaissances (chantiers, mémoires de recherche). Ce basculement entre les deux logiques, restitution vers production, est source d'une grande déstabilisation cognitive qui engendre doutes, crise de confiance, et, dans de nombreux cas, abandon (Ravestein, 2006).

L'usage d'une liste de diffusion en parallèle d'une formation présentielle est-il un bon moyen d'atténuer les effets de ce basculement? Nous espérons le montrer dans l'analyse qui suit. Le corpus brut, nettoyé des traces automatiques redondantes à chaque expédition générées par les logiciels de courriel (de, à, $\mathrm{x}$ a écrit, etc.), s'élève à 20071 mots. Nous avons procédé à une deuxième soustraction en supprimant du corpus les mots formant des formules de politesse coutumières comme «bonjour, $@+$, cordialement, etc. ». Nous avons alors soumis le corpus restant à un logiciel de tri et de comptage d'occurrences ${ }^{7}$. Celui-ci retranche à la demande les motsoutils du discours qui ne sont pas chargés de signification comme les articles, les prépositions etc. Le corpus restant se décrit, après cette opération, selon le tableau suivant :

\begin{tabular}{|c|c|}
\hline Formes $(\mathrm{F})$ & 3084 \\
\hline Occurrences $(\mathrm{O})$ & 7956 \\
\hline $\mathrm{F} / \mathrm{O}$ & $38,76 \%$ \\
\hline Hapax $(\mathrm{H})$ & 1866 \\
\hline $\mathrm{H} / \mathrm{F}$ & $60,51 \%$ \\
\hline
\end{tabular}

Tableau 1. Forme générale du corpus

On voit dès à présent que les échanges constituent un corpus qui correspond à un discours standard, avec un rapport usuel ordinaire des formes, occurrences et hapax, la concentration est normale. Nous pouvons l'affirmer d'autant plus que le corpus est conforme à la loi de Zipf.

Comme nous l'avons annoncé plus haut, nous avons construit ensuite des catégories d'analyse ad hoc pour rendre raison du corpus que nous avons retravaillé

7. Logiciel « dico» libre de droits téléchargeable sur: http://www.up.univ-mrs.fr/ veronis/ logiciels/index.html 
à l'aide d'un autre logiciel qui examine le contexte d'apparition des termes dans le discours $^{8}$.

Une première catégorie concerne les lemmes exprimant la notion de temporalité, comme les dates ou « bientôt, déjà, demain, quand, etc. ». Ils représentent 4,07 \% du discours et sont très associés aux tournures exprimant le doute où l'on retrouve des lemmes comme « éventuel, confirmer, possible, etc. ».

Une deuxième classe est constituée du vocabulaire théorique qui renvoie au travail conceptuel qui fait partie de cette formation. Nous avons été relativement surpris par la faible proportion qu'il occupe dans les échanges (1,67\%), comme si une règle tacite de la communauté en ligne partageant la liste de diffusion pratiquait une certaine censure de langage sur les objets de savoirs en jeu dans la formation. On peut faire l'hypothèse que ce vocabulaire n'est pas encore maîtrisé de la part des participants, sachant que nombre d'entre eux viennent du secteur de l'entreprise et sont en reprise d'études.

Une troisième catégorie compte les lemmes relatifs au vocabulaire technique du multimédia, qui est au cœur de cette formation. Ici, on retrouve une proportion attendue : 9,39\% du discours. Les lemmes sont associés de manière importante à la résolution de problèmes techniques qui font l'objet de demandes par les uns et de solutions par les autres. On repère donc une association de ce vocabulaire avec une certaine relation d'aide.

Nous avons également isolé les occurrences nominatives : prénoms et noms des participants, elles représentent $1,16 \%$ du corpus.

Une dernière catégorie a fait l'objet de notre découpage: les lemmes qui expriment les relations a-institutionnelles, la convivialité, etc. Ils représentent une part non négligeable du discours, soit 10,68\%.

$73,03 \%$ du discours restant est constitué des lemmes nécessaires à l'expression usuelle d'une conversation ordinaire.

Nous avons ensuite examiné les fréquences d'apparition des lemmes afin de se rendre compte d'une éventuelle concentration sur certains d'entre eux qui deviendraient ainsi porteurs d'une signification particulière relative à la teneur des échanges. Nous avons choisi de présenter les lemmes de fréquence $>20$ dans le tableau ci-après. Ils concentrent $13 \%$ des échanges.

8. Logiciel « contexte» téléchargeable sur: http://www.up.univ-mrs.fr/ veronis/logiciels/ index.html 


\begin{tabular}{|l|c|c|}
\hline \multicolumn{1}{|c|}{ Lemmes très fréquents } & Occurrences & $\begin{array}{c}\text { Fréquences } \\
\text { (en \%) }\end{array}$ \\
\hline chantier & 125 & 12,1 \\
\hline message & 78 & 7,6 \\
\hline groupe & 70 & 6,8 \\
\hline former & 68 & 6,6 \\
\hline voir & 58 & 5,6 \\
\hline proposer & 53 & 5,2 \\
\hline stage & 48 & 4,7 \\
\hline travail & 47 & 4,6 \\
\hline semaine & 43 & 4,2 \\
\hline penser & 42 & 4,1 \\
\hline intérêt & 40 & 3,9 \\
\hline idée & 38 & 3,7 \\
\hline répondre & 38 & 3,7 \\
\hline sujet & 33 & 3,2 \\
\hline chercher & 28 & 2,7 \\
\hline mémoire & 27 & 2,6 \\
\hline question & 27 & 2,6 \\
\hline devoir & 26 & 2,5 \\
\hline problème & 25 & 2,4 \\
\hline information & 24 & 2,3 \\
\hline comprendre & 24 & 2,3 \\
\hline espoir & $\mathbf{1 0 2 9}$ & 2,2 \\
\hline temps & & $\mathbf{1 0 0}$ \\
\hline Media & 23 & \\
\hline \multicolumn{1}{|c|}{ Total } & 22 & \\
\hline
\end{tabular}

Tableau 2. Principaux lemmes

Nous remarquons que la production écrite demandée, qui est l'enjeu de la validation et qui est désignée par le terme « chantier » dans le jargon des étudiants et des professeurs, est le terme qui revient le plus fréquemment. Ainsi, parmi les $13 \%$ d'échange, l'objet de la discussion porte bien sur la tâche à réaliser. Pour reprendre la typologie proposée par Peraya $(2000,2002)$, la dimension référentielle est la plus importante.

Si maintenant nous reprenons la typologie de nos travaux précédents (Audran et Simonian, 2003 ; Simonian et Ciussi, 2004), on s'aperçoit que la dimension sociale 
est la plus importante. Les échanges relatifs à la technique sont faibles ainsi que la dimension évaluative ; une explication possible peut être liée à une tâche orientée par une logique de production et non de restitution. En effet, le fait de ne pas proposer de contenus à travailler et à restituer sous une certaine forme autorise les étudiants à se positionner en tant que producteurs de savoirs plutôt qu'en tant que lecteurs. L'interaction entre les étudiants semble liée clairement au travail de production. Cependant, les échanges ne se situent pas dans le champ de la réalisation de la tâche mais dans celui de l'élaboration et de la construction collective des méthodes de sa réalisation. De ce point de vue, il semble que la construction des connaissances s'exerce de manière collaborative et négociée (Stahl, 2002). Le modèle socioconstructiviste devient ici tout à fait opérant pour expliquer la coopération et surtout la communication réflexive que nous observons (Engeström, 1987). La liste de diffusion semble permettre, dans le cadre d'un travail de production, de donner naissance à une " communauté d'apprenant » à travers des échanges axés davantage sur la manière de faire que sur des concepts.

Ainsi, les étudiants intéragissent davantage sur la dimension réflexive du faire (création et faisabilité) que sur la dimension théorique et conceptuelle du faire.

Ces résultats nous permettent d'identifier des différences avec les travaux effectués précédemment, dans lesquels les étudiants étaient placés dans un travail de restitution (Audran et Simonian, 2003 ; Simonian et Ciussi, 2004). Ces différences peuvent s'expliquer par le fait qu'il n'y a pas d'échanges à partir d'un contenu ou d'un cours. Un travail de production semble conduire les étudiants à tenir des échanges s'inscrivant dans une dimension collaborative et non hiérarchisée et dans une dimension horizontale syntagmatique (Audran, 2001). En effet, la concentration de lemmes renvoyant à une dimension réflexive sur la tâche est une caractéristique de ce corpus.

\section{Conclusion}

Inviter des étudiants à utiliser une liste de diffusion pour s'entraider à réaliser une tâche prescrite qui doit aboutir à une production originale, semble favoriser la dimension collaborative de l'outil de communication.

Lorsqu'on travaille plus traditionnellement dans une logique pédagogique orientée vers la bonne restitution dans les formes convenables de contenus académiques, l'usage de la liste de diffusion est bien différent et l'aspect collaboratif est bien moins présent (Audran et Simonian, 2003 ; Simonian et Ciussi, 2004).

Par conséquent, ce n'est pas seulement en désignant a priori un outil de communication comme outil de collaboration dans une ingénierie didactique que les usagers vont s'en servir comme on le souhaite, il faut également que l'objet des échanges soit approprié et corresponde à la situation souhaitée (Audran, souspresse). La centration du vocabulaire des échanges autour de l'objet et de la manière de réaliser la tâche montre bien qu'un objet d'apprentissage imaginé dans une 
logique de production favoriserait effectivement l'utilisation de la liste de diffusion comme outil de collaboration.

Si les étudiants qui entrent dans des cursus de recherche après leurs études de base rencontrent des difficultés pour adopter une logique de production de savoirs, les outils comme la liste de diffusion pourraient donc atténuer la déstabilisation cognitive, les doutes, la crise de confiance qui, souvent, génèrent des abandons.

L'usage d'une liste de diffusion peut constituer une aide à l'apprentissage tout en restant marginalement un moyen d'exprimer librement doutes et difficultés, un lieu de construction de liens conviviaux; comment? En devenant une véritable ressource (Laurillard, 1995) dans une stratégie orientée par une logique pédagogique de production de connaissances par les étudiants. C'est bien davantage dans les conditions d'une production qu'une liste de diffusion devient un véritable environnement collaboratif.

\section{Bibliographie}

Audran J., Influences réciproques relatives à l'usage des Nouvelles Technologies de l'Information et de la Communication par les acteurs de l'école. Le cas des sites Web des écoles primaires françaises, Thèse de Doctorat, Université de Provence, 2001.

Audran J., «Le dispositif ne fait pas la situation. Heurs et malheurs des formations en ligne », Charlier B. et Peraya D., Transformation des regards sur la recherche en technologie de l'éducation. Bruxelles, De Boeck (sous-presse).

Audran J. et Simonian S., «Profiler les apprenants à travers l'usage du forum», International journal of Information Sciences for Decision Marketing, $\mathrm{n}^{\circ}$ 10, 2003. http://isdm.univ-tln.fr

Barchechat E. et Pouts-Lajus S., Le design des didacticiels : guide pratique pour la conception de scénarios pédagogiques interactifs, Paris, ACL, 1990.

Bateson G., Vers une écologie de l'esprit, Paris, Editions du Seuil, 1977.

Bottino R.M., "The evolution of ICT-based learning environments with perspectives for the school of the future?", British Journal of Educational Technology, 35 (5), 2004, p. 553-567.

Choplin H., Cortesi-Grou N., Perrier F., Craipeau S., « Communauté ou réseau ? à partir d'un collectif professionnel », Communication et Langages, $n^{\circ}$ 144, 2005, p. 13-24.

Drot-Delange B., "Teachers and electronic communication: a minority activity?", Educational Media International, 38, n 2/3, 2001, p. 141-148.

Dubour X., Gounon P., Leroux P., «Décrire l'accompagnement des apprenants », Environnements Informatiques pour l'Apprentissage Humain, 2005, p. 261-272.

Engeström Y., Learning by Expanding. An activity-theoretical approach to developmental research, Helsinky, Orienta, Konsultit, 1987.

Engeström Y., "Expertise as Mediated Collaborative Activity”, Interactive Expertise: Studies in Distributed Working Intelligence, University of Helsinki Department of Education Research Bulletin, n 83, 1992. 
Fry P. et Coe K., "Interactions among dimensions of academic motivation and classroom social climate: a study of the perceptions of junior high and high school students", British Journal of Educational Technology, $\mathrm{n}^{\circ}$ 50, 1980, p. 33-42.

Goffman E., Façons de parler, Paris, Editions de Minuit, 1987.

Harasim L., Hiktz R., Teles L. et Turoff M., Learning networks. A files guided to teaching and learning on line, MIT Press, Cambridge, Massachusetts, 1995.

Henri F. et Lundgren Kayrol K., Apprentissage collaboratif à distance, Montréal, PUQ, 2001.

Henri F. et Pudelko B., «La recherche sur la communication asynchrone : de l'outil aux communautés », Daele A. et Charlier B., Les communautés délocalisées d'enseignants, Paris, Publication de la Maison des Sciences de l'Homme, 2002.

Hoyles C., Healy L. et Pozzi S., "Interdependence and autonomy: aspects of groupwork with computers", Learning and instruction, $\mathrm{n}^{\circ}$ 2, 1992, 239-257.

Hoyles C., Healy L. et Pozzi S., "Groupwork with computers: an overview of findings", Journal of Computer Assisted Instruction, $\mathrm{n}^{\circ}$ 10, 1994, p. 202-215.

Jacquinot G., «L'interactivité, Dernier des postulats fondamentaux... et menteurs ! », La Lettre d'ATENA, n 27, 1996, p. 8.

Johson D., Johson R. et Stanne M., "Comparison of computer-assisted cooperative, competitive and individualistic learning", American Educational Research Journal, n 23 (3), 1986, p. 382-392.

Kaye A., Collaborative learning through computer conferencing: the najaden paper, NewYork, Springler Verlag, 1992.

Kopper R., Learning Design, 2003, http:/www.imsglobal.org/learningdesign/

Lamirault O., «IAE de Caen : apprentissage collaboratif dans l'e-formation », Actualité de la Formation permanente, $\mathrm{n}^{\circ} 179,2002$, p. 74-78.

Laurillard D., "Multimedia and the changing experience to learner", British Journal of Educational Technology, $\mathrm{n}^{\circ} 26$ (3), 1995, p. 179-189.

Linard M., «Les Tic en éducation : un pont possible entre faire et dire», Les jeunes et les médias, Paris, Hachette, 2000, p. 151-177.

Mondada L., «Formes de séquentialité dans les courriels et les forums de discussion », $A L S I C, \mathrm{n}^{\circ} 2$ (1), 1999, p. 3-25.

Myrdal S., "Teacher Education on-line: what guest lost in electronic communication?", Educational Media International, $\mathrm{n}^{\circ} 31$ (1), 1994, p. 46-52.

Peraya D., «Théorie de la communication et technologies de l'information et de la communication. Un apport réciproque», Revue européenne des sciences sociales, XXXVI, nº 111, 1998, p. 171-188.

Peraya D., Projet Poshiavo. Rapport final du sous-projet 2, 2000, http://tecfa.unige. $\mathrm{ch} /$ tecfa/research/poschiavo/ligne2/ 
Peraya D. et Dumont P., "Teletutorials and virtual calssrooms: is it possible to avoid forming habits?", Fluckiger F., Jutz C., Schltz P. et Cantoni L. (eds), $4^{\text {th }}$ international conference on neweducational environment, 2002, p. 8-11.

Ravestein J., Contribution à une anthropologie didactique des technologies de l'information et de la communication en éducation, HDR,Université de Provence, 2006.

Ravestein J. et Simonian S., «Etude de la constitution d'un groupe virtuel en formation à distance ", Actes du $2^{e}$ colloque international de Guéret, 2003, Cédérom.

Sensevy G., Institutions didactiques: étude et autonomie à l'école élémentaire, Paris, Presses universitaires de France, 1998.

Simonian S. et Ciussi M., "L'échange favorisé par l'organisation relationnelle du contenu », International journal of Information Sciences for Decision Marketing, $\mathrm{n}^{\circ}$ 18, 2004. http:// isdm.univ-tln.fr

Slavin R., "Classroom reward structure: an analytical and pratical review", Review of Educational Research, $\mathrm{n}^{\circ} 47$ (4), 1977, p. 633-650.

Stahl G., Computer Support for Collaborative Learning: Foundations for a CSCL Community, Lawrence Erlbaum, Boulder, 2002.

Teasley S.D. et Roschelle J., "Constructing a joint problem space: The computer as a tool for sharing knowledge", Lajoie S.P. et Derry S.J. (dir.), Computers as cognitive tools, Hillsdale (NJ): Lawrence Erlbaum Associates, 1993, p. 229-258.

Viens J. et al., Rapport de recherche du collectif sur l'Apprentissage Collaboratif à l'aide des TIC (CRACTIC), 2001.

Zack M.H., "Using electronic massaging to improve the quality of instruction", Journal Education of Business, $\mathrm{n}^{\circ} 70$ (4), 1995, p. 202-206. 